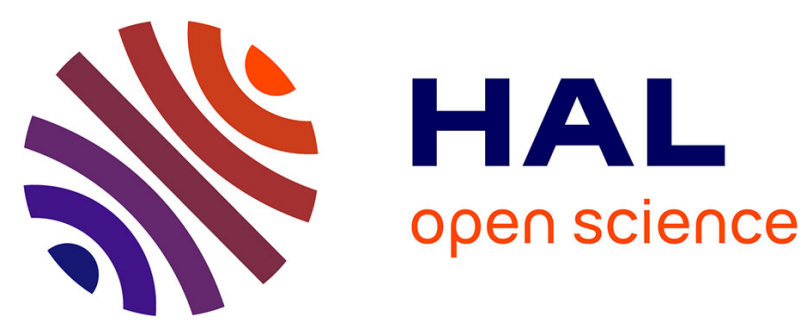

\title{
Detection of Multiple Pathways in the Spinal Cord White Matter Using Q-Ball Imaging
}

Julien Cohen-Adad, Maxime Descoteaux, S. Rossignol, R. D. Hoge, Rachid Deriche, Habib Benali

\section{To cite this version:}

Julien Cohen-Adad, Maxime Descoteaux, S. Rossignol, R. D. Hoge, Rachid Deriche, et al.. Detection of Multiple Pathways in the Spinal Cord White Matter Using Q-Ball Imaging. Biomedical Imaging: From Nano to Macro, 2008. ISBI 2008. 5th IEEE International Symposium on, May 2008, Paris, France. pp.696-699, 10.1109/ISBI.2008.4541091 . inria-00423368

\section{HAL Id: inria-00423368 https://hal.inria.fr/inria-00423368}

Submitted on 19 Nov 2009

HAL is a multi-disciplinary open access archive for the deposit and dissemination of scientific research documents, whether they are published or not. The documents may come from teaching and research institutions in France or abroad, or from public or private research centers.
L'archive ouverte pluridisciplinaire HAL, est destinée au dépôt et à la diffusion de documents scientifiques de niveau recherche, publiés ou non, émanant des établissements d'enseignement et de recherche français ou étrangers, des laboratoires publics ou privés. 


\title{
DETECTION OF MULTIPLE PATHWAYS IN THE SPINAL CORD WHITE MATTER USING Q-BALL IMAGING
}

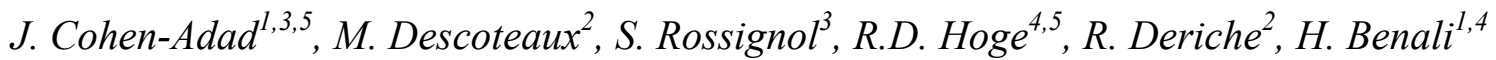 \\ ${ }^{1}$ INSERM U678, Université Pierre et Marie Curie (Paris VI), CHU Pitié-Salpêtrière, Paris, France \\ ${ }^{2}$ Odyssée Project Team, INRIA/ENPC/ENS, INRIA Sophia Antipolis, France \\ ${ }^{3}$ GRSNC, Faculty of Medicine, Université de Montréal, Montreal, QC, Canada \\ ${ }^{4}$ Unité de Neuroimagerie Fonctionnelle, CRIUGM, Université de Montréal, Montreal, QC, Canada \\ ${ }^{5}$ Institute of Biomedical Engineering, Université de Montréal, Montreal, QC, Canada
}

\begin{abstract}
High angular resolution MRI such as q-ball imaging (QBI) allows to recover complex white matter architecture. We applied this technique to an ex vivo spinal cord of one cat using a $3 \mathrm{~T}$ scanner, 100 directions and b-values varying from 1000 to $3000 \mathrm{~s} / \mathrm{mm}^{2}$. As a result, QBI can retrieve crossing fibre information, where the diffusion tensor imaging approach is constrained to a single diffusion direction. To our knowledge, this is the first study demonstrating the benefits of QBI in observing longitudinal, commissural and dorso-ventral fibres in the spinal cord. It is a first step towards in vivo characterization of the healthy and injured spinal cord using high angular resolution diffusion imaging (HARDI) and QBI.
\end{abstract}

Index Terms - Diffusion tensor imaging, high angular resolution imaging, q-ball, spinal cord, white matter

\section{INTRODUCTION}

Diffusion tensor imaging (DTI) of the spinal cord has demonstrated its benefits for assessing white matter integrity following injury [1]. Also, it has been shown that DTI could retrieve major longitudinal pathways, i.e., axon bundles oriented in the rostro-caudal direction [2]. However, other types of fibres not oriented longitudinally are present in the spinal cord. These are for instance commissural fibres coursing in the medio-lateral direction, and dorso-ventral fibres that may originate from dorsal root afferents or descending tracts. Since the diffusion tensor can only account for a single major diffusion direction and, to a certain extent, for a minor diffusion through the second eigenvector (see Discussion), these pathways might not be visible using DTI.

To overcome this issue, model-free approaches have been proposed to measure the microscopic diffusion without constraining its representation. These methods are known as q-space imaging or diffusion spectrum imaging and have already demonstrated benefits, notably for imaging the spinal cord [3]. However, long acquisition time is required for an efficient sampling of the q-space to retrieve the threedimensional diffusion profile. For that purpose, a similar method has been proposed which consists in sampling the qspace restricted to a single sphere. This method, also known as q-ball imaging (QBI), allows to retrieve crossing fibres information with shorter acquisition time [4].

In the present study, we applied this technique to an $e x$ vivo spinal cord and we show that $\mathrm{QBI}$ can not only retrieve longitudinal pathways as DTI does, but it can also represent medio-lateral and dorso-ventral directions which are consistent with the known architecture of the spinal cord.

\section{MATERIAL AND METHODS}

\subsection{Ex vivo spinal cord of cat}

The experiments were conducted in accordance with the Ethics Committee of the University of Montreal. One cat was sacrificed with an overdose of barbiturate (Somnotol). A laminectomy was performed at the lumbar level followed by the dissection of the dura matter. Dorsal and ventral roots were sectioned. The cord corresponding to lumbar segments 3 to 7 was extracted and put in saline solution (0.9\%). 30 minutes after extraction, the cord was placed into a gelatine solution (6\%). It was imaged 5 hours later.

\subsection{MRI acquisition}

Experiences were conducted on a Siemens 3T scanner using a receive-only 8-channels spine array coil to get the benefits of parallel imaging. Diffusion-weighted (DW) scans were acquired with a single-shot spin echo EPI pulse sequence using the same strategy as in [2, 5] for reducing susceptibility artefacts and eddy-currents. Parameters were: matrix $=120 \times 120$, voxel size $=1 \times 1 \times 3 \mathrm{~mm}^{3}, \mathrm{TR}=4000 \mathrm{~ms}$, $\mathrm{TE}=96 \mathrm{~ms}$, flip angle $=90^{\circ}, 3$ repetitions, b-value $=1500$ $\mathrm{s} / \mathrm{mm}^{2}, 100$ directions using a polyhedron scheme. Three 

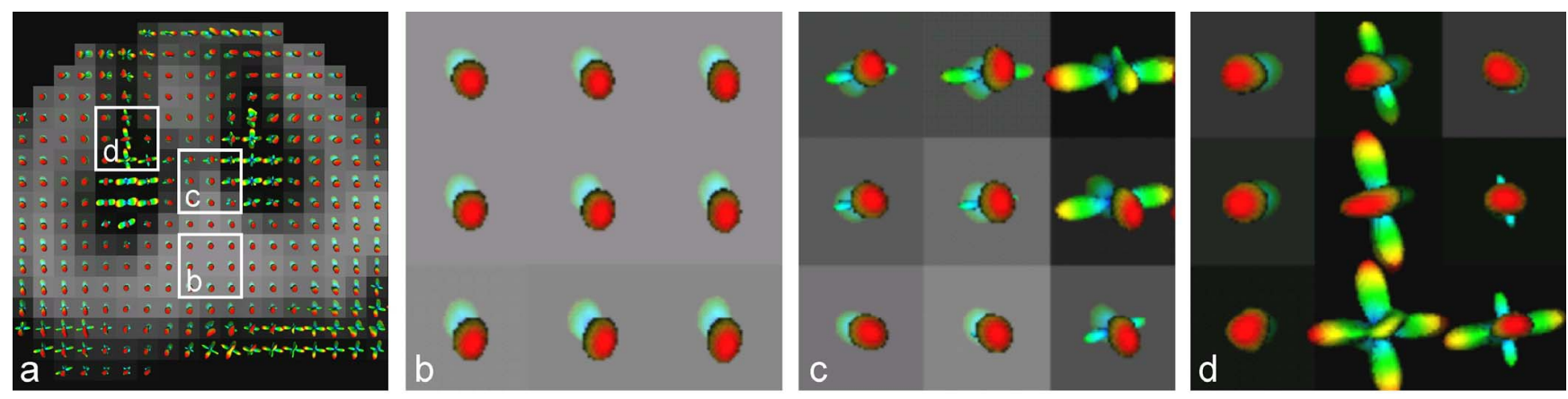

Fig. 1. Results of QBI in the spinal cord. FODFs were overlaid on an axial GFA map (a, top is dorsal). Zoomed panels show fODFs in the ventral white matter (b), in the central canal (c) and in the right grey matter posterior horn (d).

additional data were acquired using a transmit-receive wrist coil to get higher signal-to-noise ratio. No parallel imaging was used for these scans. The objective was to compare different b-values. Parameters were: matrix $=128 \times 128$, voxel size $=0.65 \times 0.65 \times 3 \mathrm{~mm}^{3}, \mathrm{TR}=4700 \mathrm{~ms}, \mathrm{TE}=152$ ms, flip angle $=90^{\circ}, 3$ repetitions, $b$-value $=1000,2000$ and $3000 \mathrm{~s} / \mathrm{mm}^{2}, 100$ directions.

Anatomical scans were conducted using proton-density weighting to obtain good distinction between white and grey matter. Parameters were: Turbo Spin-Echo sequence (turbo factor 13), matrix $=320 \times 320$, voxel size $=$ $0.25 \times 0.25 \times 3 \mathrm{~mm}^{3}, \mathrm{TR}=3500 \mathrm{~ms}, \mathrm{TE}=11 \mathrm{~ms}$, flip angle $=$ $120^{\circ}$.

\subsection{Q-ball estimation}

QBI, originally proposed in [4], reconstructs the diffusion orientation distribution function (dODF) directly from the raw high angular resolution diffusion imaging (HARDI) measurements on a single sphere using the Funk-Radon transform (FRT). This FRT can be solved analytically, efficiently and robustly with the spherical harmonic (SH) basis [6]. The key idea is to express the HARDI signal as a SH series of order $L$ and to solve the FRT using the FunkHecke theorem. The final ODF reconstruction, $\Psi$, in direction $(\theta, \varphi)$ is

$$
\Psi(\theta, \varphi)=\sum_{k=0}^{L} \sum_{m=-k}^{k} 2 \pi P_{k}(0) c_{k}^{m} Y_{k}^{m}(\theta, \varphi)
$$

where $Y_{k}^{m}$ denote SH of order $k$ and degree $m, c_{k}^{m}$ are the SH coefficients describing the input HARDI signal and $P_{k}$ is a Legendre polynomial of order $k$. Note that it is also possible to impose a Laplace-Beltrami regularization criterion while estimating the SH coefficients $c_{k}^{m}$, as done in [6]. Here, we used this regularization and estimation order $L$ $=4$ to get robust fibre detection. From the dODF reconstructed, we computed the generalized fractional anisotropy (GFA) [4], which is a HARDI anisotropy measure similar to the popular DTI fractional anisotropy (FA). The dODF is a blurred version of the "true" fibre ODF (fODF). Because of this blurring effect, extracted maxima of the dODF are often used for fibre analysis. An alternative is to use spherical deconvolution methods that provide a direct estimate of the fODF [7]. These techniques have better angular resolution than dODF and produce sharper fODF profiles [8]. Thus, small fibre compartments with small volume fractions are sometimes visible with the fODF and not with the dODF.

\subsection{Data processing}

Images were first averaged respectively to their diffusion directions and were two times interpolated. Series acquired without parallel imaging were corrected for susceptibilityinduced distortions using the method described in [2]. Diffusion tensors (DTs), dODFs and fODFs were estimated using the same method as in $[6,8]$. Based on the anatomical scan, a manual segmentation was performed to delineate white matter from grey matter over 10 axial slices. Two masks were then created. FA and GFA were computed respectively from DTs and dODFs, and were averaged within each created mask.

\section{RESULTS}

\subsection{Observation of multiple pathways}

After processing the data and extracting ODF maxima, a major diffusion direction was almost exclusively observed in the white matter along the rostro-caudal direction (Fig. 1b). This result was expected since major ascending and descending fibres follow such an orientation. More interestingly, other directions were observed within the spinal cord grey matter as described hereafter.

A medio-lateral direction was obtained in the central canal and might correspond to commissural fibres (Fig. 1c). These axons, linking both sides of the spinal cord grey matter, notably originate from grey matter interneurons and cross to the opposite side through the anterior commissure.

A dorso-ventral direction was obtained throughout the grey matter (Fig. 1d). It might correspond to fibres arising from the projection of dorsal roots (sensory afferents) at several spinal levels. 


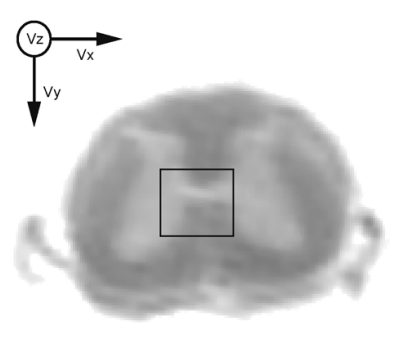

a
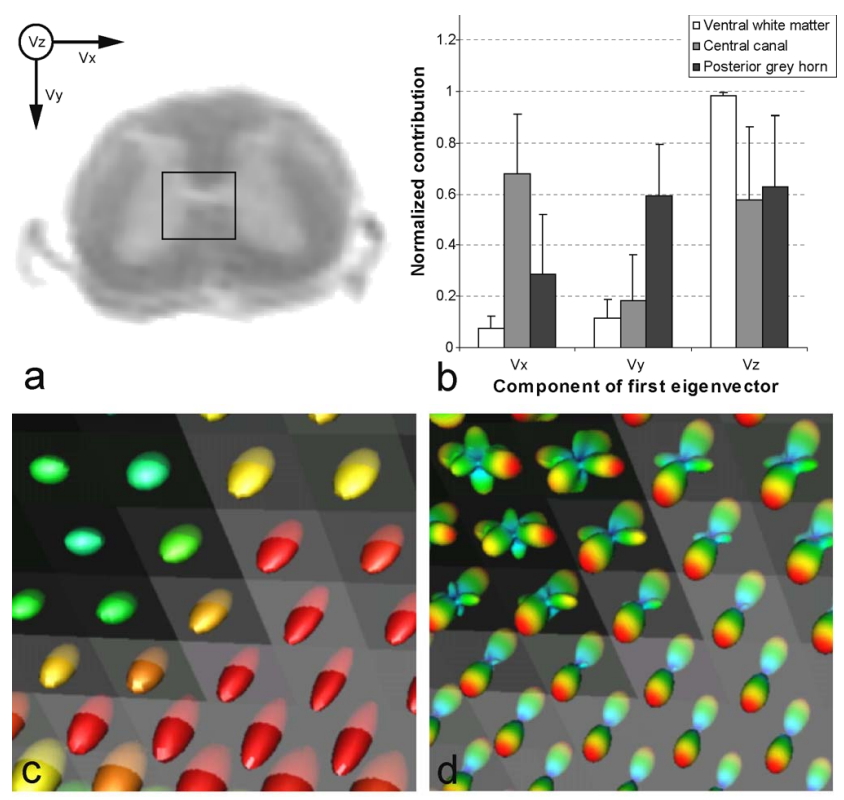

Component of first eigenvector

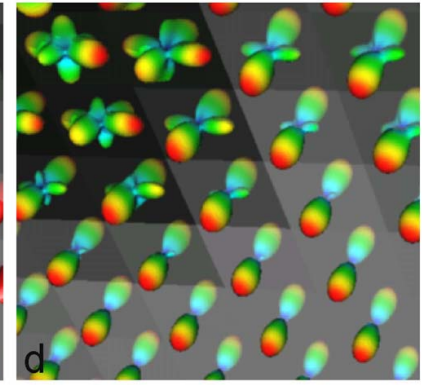

Fig. 2. Comparison between DTI and QBI. Axial anatomical MRI with a selected region including the white matter, the central canal and partially the dorsal and ventral horn (a). Components of diffusion tensors first eigenvector in three different regions of the cord (b). Overlay of DTs (c) and fODFs (d) for the selected region of interest over a GFA axial slice.

\subsection{Comparison between DTI and QBI}

To qualify the benefits of QBI over DTI, we compared both approaches by selecting a region including the three major pathways that were observed previously, i.e., longitudinal, medio-lateral and dorso-ventral. As expected, tensors yielded very anisotropic profile in the white matter, pointing in the longitudinal direction (red ellipsoids in Fig. 2c bottom-right). Similarly, fODFs presented one unique direction (Fig. 2d bottom-right). The almost unique component along the cord was confirmed by looking at tensors' first eigenvector which stood almost exclusively along the cord axis (Vz in Fig. 2b).

However, in the grey matter central canal the disk shape of tensors suggested two first eigenvalues of the same order (yellow ellipsoids in Fig. 2c top-right). Quantification of tensors first eigenvector components indeed showed an increase of water diffusion in the lateral direction ( $\mathrm{Vx}$ in Fig. 2b). In the case of fODFs, one can clearly distinguish these two majors diffusion directions (Fig. 2d top-right).

When looking at a region more lateral than that in the central canal, tensors tend to become spherical indicating an apparent loss of FA without one clear diffusion direction. This region of the grey matter is known to be the site of intermingled fibres coursing in various directions. However, longitudinal and lateral pathways are still present. It resulted in first eigenvectors having components in the three directions (Fig. 2b). This intravoxel heterogeneity was better described by fODFs which pointed in the longitudinal, lateral and antero-posterior directions (Fig. 2d top-left).

\subsection{Impact of $b$-value}

The choice of $b$-value is related to the attenuation of moving protons in each of the diffusion weighting directions. Small b-values produce high signal but low angular resolution. On the contrary, large $b$-values provide better angular resolution but MRI signal recorded from moving protons is more attenuated, yielding lower signal. The b-value should then be carefully set regarding the q-space sampling.

In that study, we did three acquisitions at 100 directions using b-values of 1000,2000 and $3000 \mathrm{~s} / \mathrm{mm}^{2}$. Results showed that with such a high sampling rate, low b-values yielded maxima oriented in the dorso-ventral direction, which might be inconsistent when observing the central canal region (Fig. 3b). Indeed, no fibres are known to course in the dorso-ventral direction at that precise location. On the contrary, higher b-values decrease the number of maxima in the area of commissural fibres (Fig. 3d).

GFA quantifications showed lower apparent anisotropy at lower b-values, in both the white and the grey matter (Tab. 1). That result raises the question of accuracy in reporting FA values computed in a region that is prone to crossing fibres.

\begin{tabular}{ccc}
\hline B-value & White matter & Grey matter \\
\hline 1000 & $0.32 \pm 0.16$ & $0.14 \pm 0.04$ \\
\hline 2000 & $0.54 \pm 0.15$ & $0.23 \pm 0.05$ \\
\hline 3000 & $0.73 \pm 0.05$ & $0.27 \pm 0.04$
\end{tabular}

Tab. 1. GFA quantification in the white and grey matter at various b-values (given in $\mathrm{s} / \mathrm{mm}^{2}$ ).

\section{DISCUSSION}

\subsection{Benefits of QBI for the spinal cord}

As demonstrated in this study, QBI can retrieve crossing fibre information in an ex vivo spinal cord, whereas the DTI approach is constrained by its single diffusion direction. Although the second eigenvector might be used to account for crossing fibres in the brain [9] and in the spinal cord [10], there is a restriction imposed by the tensor itself. When the primary direction is defined by the principal eigenvector, the second eigenvector is limited in degrees of freedom since its direction is necessary on the plane orthogonal to longitudinal fibres. In the presence of non orthogonal fibres, the usual way of decomposing the tensor becomes less efficient. Moreover, in case of intravoxel heterogeneity one cannot easily rely on the first eigenvector's orientation since the latter results from a linear combination of major diffusion directions. Knowing that spinal cord architecture is not only oriented rostro- 


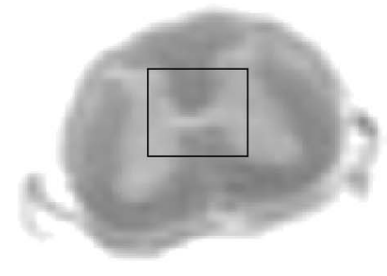

a
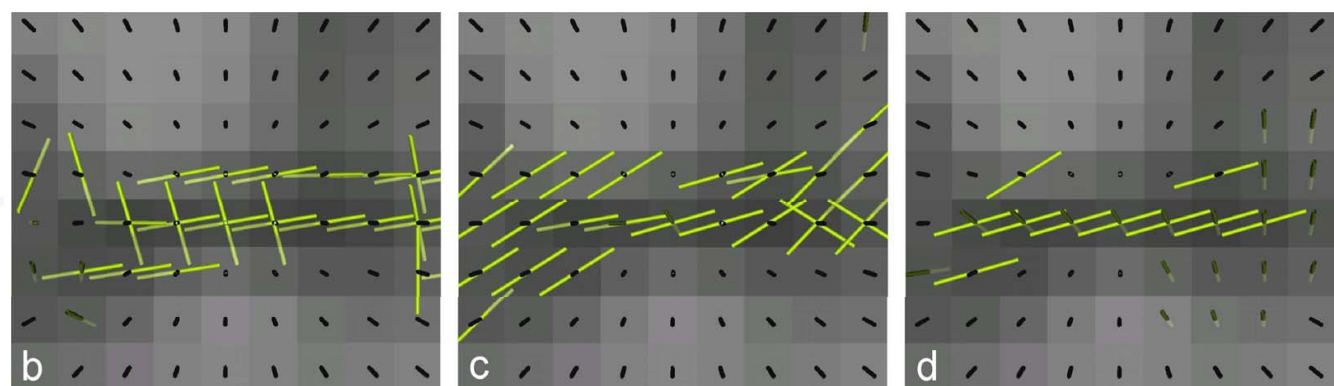

Fig. 3. Impact of b-value on fODF estimation. Comparison of fODF maxima in a region centred over the central canal (a) for bvalues of $1000(\mathrm{~b}), 2000$ (c) and $3000 \mathrm{~s} / \mathrm{mm}^{2}$ (d). Maxima are overlaid on a GFA map estimated from data at $3000 \mathrm{~s} / \mathrm{mm}^{2}$.

caudal, HARDI techniques combined with QBI could be an efficient tool aiming at representing this subtle intravoxel heterogeneity.

\subsection{Strategy for optimal b-value}

Results showed more extracted maxima at lower b-values. However, some maxima might have not been consistent with the known architecture of the spinal cord's axonal pathways, such as dorso-ventral directions within the central canal region. These preliminary results suggest that low bvalues increase the sensitivity of retrieving diffusion directions but reduce their specificity. Further studies are required for optimizing such sensitive parameters in HARDI acquisition.

\subsection{Perspectives}

The next step will be to validate the method by in vivo acquisitions. One application of the technique would be the study of white matter reorganization following spinal cord injury. Indeed, there may be regeneration of damaged axons or collateral sprouting of axons in spared descending and ascending pathways or primary afferents. These new growths can project through different spinal paths as they are forming new circuits and adopt unpredicted trajectories as they by-pass the scar tissue [11]. Such a subtle reorganisation might be visible using QBI methods.

\section{ACKNOWLEDGMENTS}

We thank H. Leblond for the extraction of the spinal cord, and M. Gendron for his advices on gelatine preparation. This work was supported by the Canada Research Chair on the Spinal Cord to S.R. (CIHR) and by the Multidisciplinary Team on Locomotor Rehabilitation (CIHR). J.C.-A. has a fellowship from the Fondation pour la Recherche Médicale.

\section{REFERENCES}

[1] D. Lammertse, D. Dungan, J. Dreisbach, S. Falci, A. Flanders, R. Marino, and E. Schwartz, "Neuroimaging in traumatic spinal cord injury: an evidence-based review for clinical practice and research," $J$ Spinal Cord Med, vol. 30, pp. 205-14, 2007.

[2] J. Cohen-Adad, H. Benali, R. D. Hoge, and S. Rossignol, "In vivo DTI of the healthy and injured cat spinal cord at high spatial and angular resolution," Neuroimage, vol. 40, pp. 685-697, Apr 12008.

[3] Y. Assaf, A. Mayk, and Y. Cohen, "Displacement imaging of spinal cord using q-space diffusionweighted MRI," Magn Reson Med, vol. 44, pp. 713-22, Nov 2000.

[4] D. S. Tuch, "Q-ball imaging," Magn Reson Med, vol. 52, pp. 1358-72, Dec 2004.

[5] J. Cohen-Adad, H. Benali, and S. Rossignol, "Methodology for MR diffusion tensor imaging of the cat spinal cord," Conf Proc IEEE Eng Med Biol Soc, vol. 1, pp. 323-326, 2007.

[6] M. Descoteaux, E. Angelino, S. Fitzgibbons, and R. Deriche, "Regularized, fast, and robust analytical Qball imaging," Magn Reson Med, vol. 58, pp. 497-510, Sep 2007.

[7] B. Jian and B. C. Vemuri, "A unified computational framework for deconvolution to reconstruct multiple fibers from diffusion weighted MRI," IEEE Trans Med Imaging, vol. 26, pp. 1464-71, Nov 2007.

[8] M. Descoteaux, R. Deriche, and A. Anwander, "Deterministic and Probabilistic Q-Ball Tractography: from Diffusion to Sharp Fiber Distributions," INRIA Research Report, vol. 6273, July 2007.

[9] M. R. Wiegell, H. B. Larsson, and V. J. Wedeen, "Fiber crossing in human brain depicted with diffusion tensor MR imaging," Radiology, vol. 217, pp. 897-903, Dec 2000.

[10] H. Mamata, U. De Girolami, W. S. Hoge, F. A. Jolesz, and S. E. Maier, "Collateral nerve fibers in human spinal cord: visualization with magnetic resonance diffusion tensor imaging," Neuroimage, vol. 31, pp. 2430, May 152006.

[11] S. Rossignol, "Plasticity of connections underlying locomotor recovery after central and/or peripheral lesions in the adult mammals," Philos Trans $R$ Soc Lond B Biol Sci, vol. 361, pp. 1647-1671, 2006. 